\title{
ABCDE: Directing Student Observation During High-Fidelity Simulation
}

\author{
Josephine Seale $^{1}$ (D) A Abubakar Khan ${ }^{2} \cdot$ Barnaby Hirons $^{2} \cdot$ Colin Butchers $^{1}$
}

Accepted: 7 September 2020 / Published online: 24 September 2020

(C) The Author(s) 2020

\begin{abstract}
To encourage an active learning environment during the observation stage of high-fidelity simulation, an observation tool was created to help students recognise and record the technical and non-technical skills identified whilst watching their peers. Future work will involve quantifying any educational benefits of the tool across the medical student cohort.
\end{abstract}

Keywords High-fidelity simulation $\cdot$ Medical undergraduate $\cdot$ Observation tool $\cdot$ Active learning

\section{Innovation}

Simulation-based education has become an established means of enabling medical students to both learn and practise technical and non-technical skills in a safe environment. As a result of large student numbers, the use of simulation in an undergraduate setting typically entails students alternating between participating and observing roles.

High-fidelity simulation (Hi-Fi SIM) has been incorporated throughout the undergraduate medical curriculum at King's College London (KCL). The aims of these sessions are two-fold: to enhance student knowledge and appreciation of the use of technical and non-technical (human factors) skills in clinical situations and to provide students the opportunity to develop and practise these skills in a lowrisk environment. Evaluation of this programme has resulted in positive feedback regarding the learning opportunities

Josephine Seale

Josephine.seale@kcl.ac.uk

1 GKT School of Medical Education, Simulation and Interactive Learning Centre (SaIL), King's College London, Shepherds House, London, UK

2 Postgraduate Medical Department, Weston Education Centre, King's College Hospital, London, UK provided through active participation in the simulation. In contrast, mixed responses have been attained in relation to the observation component with the majority of students requesting a more active observer role. In addition, many students report uncertainty as to the technical and non-technical skills they are expected to look for whilst watching their peers.

Previous research on simulation based education has advocated the use of observation tools in order to encourage directed as opposed to passive observation, a situation which has been reported to result in enhanced learner outcomes for the observer [1,2]. In light of this, a unique observation tool was created which aims to foster a more active learning environment for students whilst they observe their peers. In response to student feedback, the tool also provides structured guidance on the technical and nontechnical skills frequently demonstrated in clinical practice. The resultant observation aid consists of two A4 sheets of paper with a similar A-E labelled format. The 'A-E technical skills guidance sheet' (Fig. 1) is based upon the UK Resuscitation Council's ABCDE clinical assessment of acutely unwell patients [3]. In contrast, the 'A-E non-technical skills guidance sheet' (Fig. 2) consists of a bespoke A to $\mathrm{E}$ abbreviation to categorise the skills students should be observing for during the simulation as follows: Awareness of situation, Behaving as a team, Communication, Decision-making, and Escalation. A brief description of each of these categories is provided for 


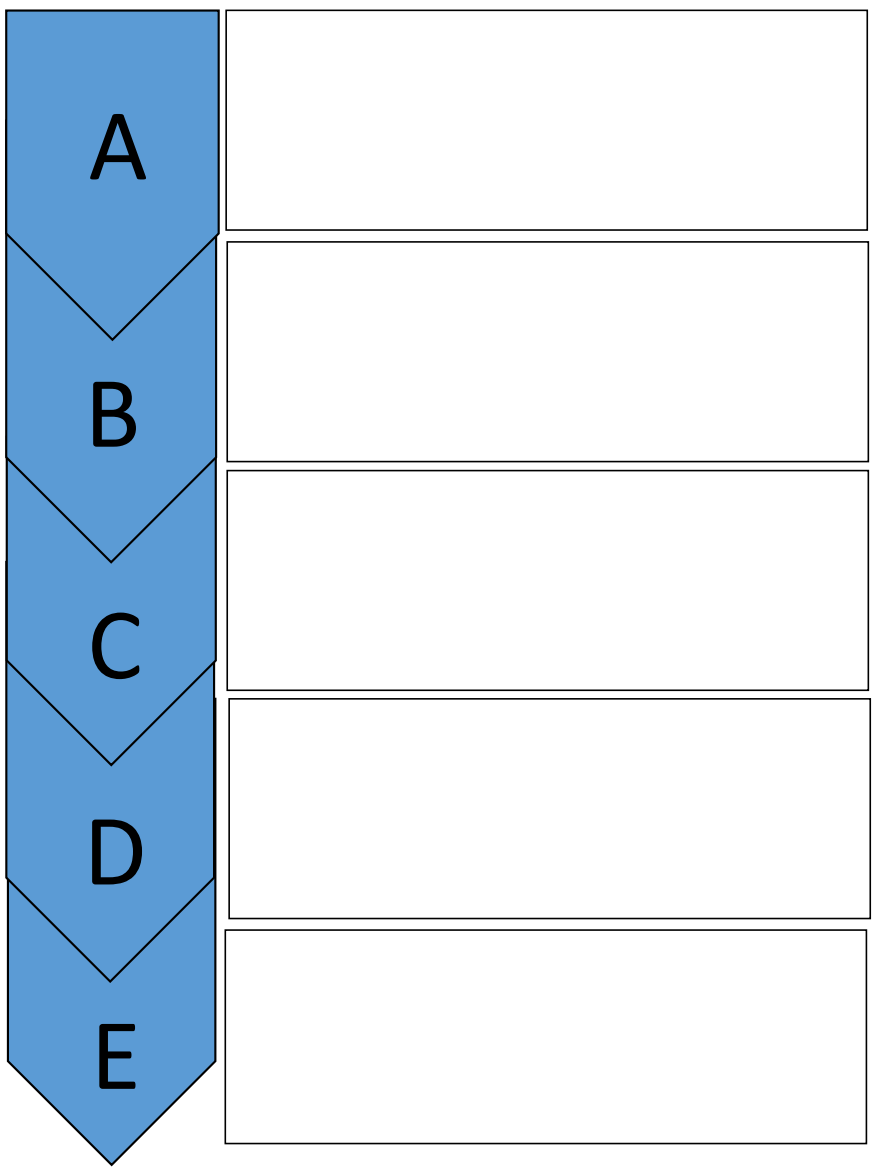

Fig. 1 A-E technical skills guidance sheet

students to refer to if needed. All non-technical skills included in the sheet are based upon those detailed in the UK General Medical Council's 'Outcomes for Graduates' [4]. Each sheet also provides space for students to record any instances in which the skills occur during the observed simulation.

The observation tool was subsequently trialled with year two KCL undergraduate medical students undergoing Hi-Fi SIM. During each half day simulation session, two medical students participated in one of four 10-min clinical scenarios involving the management of an acute clinical case focused at the level of skill expected from a year two KCL medical student. The remaining members of the group observed the scenario via a live video link. At the start of the simulation session, students were introduced to the observation tool which provided an explanation of its purpose and intended use. During each 10-min simulation, half of the observers were asked to complete an 'A-E technical skills' sheet and the other half an 'A-E non-technical skills' sheet. Students alternated between the two sheets throughout the half-day session to ensure
A-E Technical Skills Guidance Sheet (The ABCDE approach) ${ }^{1}$

AIRWAY: Look for the signs of airway obstruction.

- In complete airway obstruction, there are no breath sounds at the mouth or nose. In partial obstruction, air entry is diminished and often noisy.

- Treat airway obstruction as a medical emergency - call for expert help

BREATHING: Look, listen and feel for the general signs of respiratory distress

- Count the respiratory rate. Record the $\mathrm{SpO}_{2}$ reading of the pulse oximeter.

- Percuss and auscultate the chest. Check the position of the trachea

- All critically ill patients should be given oxygen (Note: in a subgroup of COPD patients aim for target $\mathrm{SpO}_{2}$ range of $88-92 \%$ )

CIRCULATION: In almost all medical and surgical emergencies, consider hypovolaemia to be the primary cause of shock until proven otherwise.

- Measure patient's pulse rate and blood pressure.

- Auscultate heart and look for other signs of a poor cardiac output, haemorrhage

- Unless there are obvious signs of a cardiac cause, give intravenous fluid to any patient with cool peripheries and a fast heart rate.

DISABILITY: Make a rapid initial assessment of the patient's conscious level using the AcVPU method

- Measure the blood glucose to exclude hypoglycaemia

- Common causes of unconsciousness include profound hypoxia, hypercapnia cerebral hypoperfusion or recent administration of sedatives or analgesic drugs.

EXPOSURE: To examine the patient properly full exposure of the body may be necessary. Respect the patient's dignity and minimise heat loss.

${ }^{1}$ Resuscitation Council UK. Resuscitation guidelines. 2015. Available from: https://www.resus.org.uk/resuscitation-guidelines/abcde-approach everyone had the opportunity to complete each of the sheets at least once. Every scenario was followed by a 20-min debrief with two clinically qualified tutors during which time students were encouraged to discuss and reflect on any of the skills they identified from using the AE sheets.

The observation tool was positively received by both the students and faculty involved in the simulation sessions. In particular, students commented that completing the sheets encouraged them to observe each simulation and enabled a more diverse debriefing which included both technical and non-technical factors. Tutors also identified a more in-depth analysis of the observed simulation during the debrief when compared with previous experiences without an observation tool. Following these encouraging reports, the intention is to use the observation tool as a teaching aid in all years of the KCL simulationbased undergraduate programme and to formally research whether their use translates into quantifiable differences in the acquisition of knowledge on technical and nontechnical skills. 

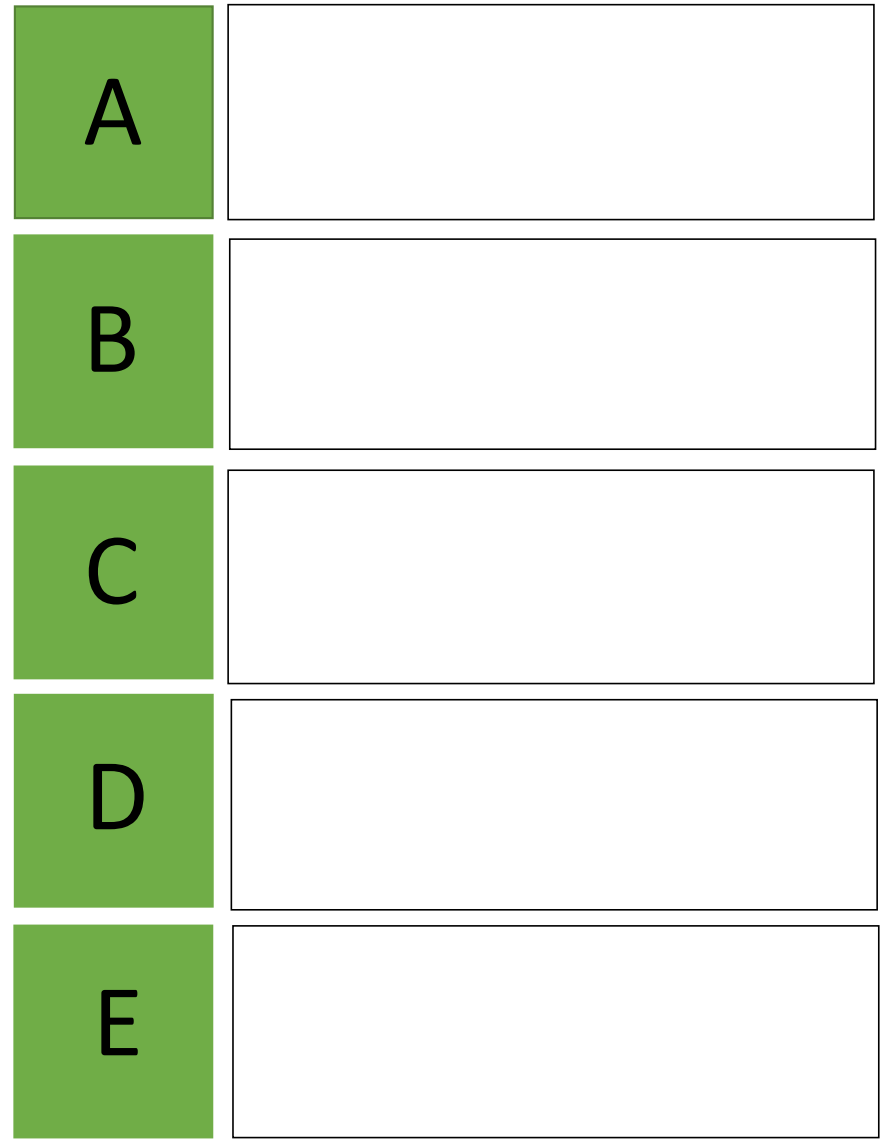

Fig. 2 A-E non-technical skills guidance sheet

Availability of Data and Material Not applicable.

Authors' Contributions Dr Seale created the A-E guidance sheets and was involved in trialling the observation tool. Dr Khan, Dr Hirons and Mr Butchers provided feedback on the observation tool and were all involved in trialling the tool.

\section{Compliance with Ethical Standards}

Conflicts of Interest The authors declare that they have no conflict of interest.

Open Access This article is licensed under a Creative Commons Attribution 4.0 International License, which permits use, sharing, adaptation, distribution and reproduction in any medium or format, as long as you give appropriate credit to the original author(s) and the source, provide a link to the Creative Commons licence, and indicate if changes were made. The images or other third party material in this article are included in the article's Creative Commons licence, unless indicated otherwise in a credit line to the material. If material is not included in the article's Creative Commons licence and your intended use is not permitted by statutory regulation or exceeds the permitted use, you will need to obtain

\section{A-E Non-Technical Skills Guidance Sheet ${ }^{\odot}$}

AWARENESS OF SITUATION

Situational awareness (SA) involves 'continuously monitoring what is happening in order to understand what is going on and what might happen in the next minutes or hours'. ${ }^{1}$ Three stages of SA can be identified:

- Student(s) notices critical cues in the environment eg. patient's vital signs

- Student(s) demonstrate an appreciation of what environmental cues mean eg. combine

information to understand the patient's current status

- Student(s) use above to anticipate what could potentially happen

\section{BEHAVING AS A TEAM}

Team-work is integral to patient safety. Some examples of team-working are:

- Student(s) demonstrate ability to work together in order to achieve a task efficiently

- Student(s) demonstrate ability to lead other members of the team and/or follow

instructions by others when required

- Workload is distributed between team members

- Information is actively shared between team members

- Student(s) guide each other if required

\section{COMMUNICATION}

Verbal and non-verbal communication skills are a vital component of ensuring patient safety. Some examples of effective communication are:

- Student(s) discuss clearly patient's current status/management/proposed plan of action - Student(s) verbalise clearly any instructions/opinions

- Closed loop communication is used to verify task completion

- Student(s) actively listen (eg. maintain eye contact, nod head etc) and respond to others

\section{DECISION MAKING}

Making decisions and the use of one's own judgement is important in order to determine the most appropriate course of action. Some examples are as follows:

- Using available information to make a decision about potential diagnosis/management

- Balancing risks and benefits of potential options

- Using own judgement to inform decisions (the impact of this will vary according to

experience and may not be evidenced in the early stages of training)

\section{ESCALATION}

Working within one's limits and subsequently calling for assistance when required is an important skill in healthcare practice. This can be demonstrated when.

- Student(s) recognise and acknowledge their own personal and professional limits

- Student(s) seek help from colleagues and supervisors where appropriate

- Student(s) handover using appropriate tool, for example SBAR

A-E Non-Technical Skills Guidance Sheet ${ }^{\circledR}$ Seale, 2019 is based on GMC Outcomes for graduate 2018 1'Murray P, Martin W Proceedings of the 10th International Symposium of AAvPA. 2012. AAvPA: Australia, permission directly from the copyright holder. To view a copy of this licence, visit http://creativecommons.org/licenses/by/4.0/.

\section{References}

1. O'Regan S, Molloy E, Watterson L, Nestel D. Observer roles that optimise learning in healthcare simulation education: a systematic review. Adv Simul. 2016;1:4.

2. Stegmann K, Pilz F, Siebeck M, Fischer F. Vicarious learning during simulations: is it more effective than hands-on training? Med Educ. 2012;46:1001-8.

3. Resuscitation Council UK. The ABCDE approach. 2015. https:// www.resus.org.uk/library/2015-resuscitation-guidelines/abcdeapproach Accessed 22 Jul 2020.

4. General Medical Council. Outcomes for graduates 2018. London: General Medical Council 2018.

Publisher's Note Springer Nature remains neutral with regard to jurisdictional claims in published maps and institutional affiliations. 